\title{
Efeito do tamanho amostral na estimativa da herdabilidade em espécies perenes
}

\author{
Marcos Deon V. de Resende* \\ Admir Lopes MorA** \\ Antonio Rioyei HigA** \\ Estefano Paludzyszyn Filho*
}

\begin{abstract}
RESUMO
No presente trabalho foram realizados estudos referentes ao tamanho amostral adequado a estimação da herdabilidade no sentido restrito em espécies perenes. Foram considerados aspectos teóricos da estimação da herdabilidade e os conceitos foram aplicados a dados experimentais associados a progênies de meios-irmãos de acácia-negra (Acacia mearnsii) avaliadas no Rio Grande do Sul. Os seguintes resultados foram obtidos: para uma precisa estimação da herdabilidade no sentido restrito recomendam-se os tamanhos amostrais aproximados de 8000, 4000 e 2600 dados para herdabilidades de 0,10; 0,20 e 0,30 respectivamente; o valor mais provável para a herdabilidade do caráter DAP em acácia-negra situa-se ao redor de 0,30; para estimação da média e variância fenotípica de um caráter quantitativo, a utilização de um tamanho amostral equivalendo a cerca de $1 / 3$ do tamanho amostral adequado à estimação da variância aditiva e da herdabilidade já é suficiente para a obtenção de estimativas precisas; com tamanho amostral inferior a 1000 não se recomenda a estimação de parâmetro genéticos. Neste caso, a predição de valores genéticos deve ser realizada preferencialmente utilizando estimativas de parâmetros relatadas em literatura.
\end{abstract}

Palavras-chave: acácia-negra; parâmetros genéticos; predição de valores genéticos; número de repetições; componentes de variância; DFREML/BLUP

\section{ABSTRACT}

Sample size effects on estimates of heritability in perennial species. This paper describes aspects concerning to optimal sample size required for

* Pesquisador da Embrapa/ CNPFlorestas

** Universidade Federal do Paraná -UFPR 
estimation of the strict sense heritability in perennial species. Theoretical concepts were applied to experimental data from Acacia mearnsii progeny test grown in Rio Grande do Sul. For a precise estimation of the heritability, sample sizes of 8000, 4000 and 2600 were recommended for heritabilities of 0,$10 ; 0,20$ and 0,30 respectively. The most probable value for the heritability of the character DBH in black wattle locates about of 0,30 . For estimation of the average and phenotypic variance of a quantitative character, the use of a sample size of about $1 / 3$ of that recommended for estimation of the additive variance and of the heritability is adequate. Sample sizes below 1000 are not suitable for genetic parameters estimation. In this case, standard values of genetic parameters (obtained from literature) should be used in the breeding values prediction procedures.

Key-words: black wattle; genetic parameters; breeding values prediction; number of replications; variance components; DFREML/BLUP

\section{INTRODUÇÃO}

Estimativas confiáveis de parâmetros genéticos são necessárias nos procedimentos de predição de valores genéticos. A rigor, para a avaliação genética, necessita-se de estimativas fidedignas de parâmetros genéticos das populações-base (originais) não selecionadas e não endogâmicas.

Definida a população de referência e o parâmetro de interesse, a escolha de um delineamento ótimo para a estimação precisa dos componentes de variância genética e fenotípica deve-se basear no critério de minimização da variância da estimativa do referido parâmetro. Tal critério conduz a determinação do tamanho amostral adequado para a estimação dos parâmetros com determinada precisão, escolhida a priori. Abordagens relevantes associadas a este tema foram representadas por ROBERTSON (1959), THOMPSON (1989) e HILL (1990). Aspectos práticos da estimação de parâmetros genéticos em espécies perenes foram relatados também por RESENDE \& ROSA-PEREZ (1999).

Dentre os parâmetros populacionais, a herdabilidade é o mais importante. Não é um parâmetro constante. As estimativas de herdabilidade variam em função do caráter, do método de estimação, da diversidade na população, do nível de endogamia da população, do tamanho da amostra avaliada, do número e tipo de ambientes considerados, da unidade experimental considerada e da precisão na condução do experimento e da coleta de dados (BORÉM, 1998).

MIRANDA FILHO (1978) ressalta que as repetições em um experimento têm como finalidade permitir uma estimativa do erro 
experimental, aumentar a precisão das estimativas e aumentar o poder dos testes estatísticos. Também enfatiza que o número de repetições necessárias em um experimento pode depender de vários fatores (graus de liberdade suficiente para uma estimativa representativa do erro experimental, limitação da quantidade de sementes, de área apropriada para a experimentação, etc.). A redução do número de repetições acarreta a redução do número de graus de liberdade do resíduo, reduzindo a probabilidade de encontrar as diferenças significativas entre tratamentos e conseqüente perda na precisão do efeito do tratamento (WOOLONS, 1980).

No melhoramento de espécies perenes, os estudos envolvendo o número adequado de repetições e plantas por parcela na experimentação têm enfatizado a detecção de diferenças significativas entre tratamentos (WRIGHT \& FREELAND, 1960; LEE, 1983; LAMBETH et al., 1983; COTERILL \& JAMES, 1984 e HAAPANEN, 1992), a eficiência na seleção de progênies (CASTRO, 1992) e de indivíduos, genitores e clones (RESENDE, 1995). Por outro lado, os estudos enfatizando o tamanho amostral para a estimação de parâmetros genéticos são escassos.

Neste trabalho são enfocados os aspectos teóricos do tamanho amostral adequado para a estimação da herdabilidade e os conceitos são aplicados a dados experimentais associados a progênies de meios-irmãos de Acacia mearnsii De Wild. avaliadas no Rio Grande do Sul, com vistas a inferências sobre a amostragem a ser praticada nas diferentes situações.

\section{MATERIAL E MÉTODOS}

\section{Minimização da variância da estimativa da herdabilidade}

A variância da estimativa da herdabilidade obtida com base na covariância de meios-irmãos equivale a (ROBERTSON, 1959):

$\operatorname{Var}\left(\mathrm{h}^{2}\right)=32\left[1+(\mathrm{N}-1) \mathrm{h}^{2} / 4\right]\left(1-\mathrm{h}^{2} / 4\right)^{2} /[\mathrm{N}(\mathrm{N}-1)(\mathrm{p}-1)]$ ou aproximadamente $\operatorname{Var}\left(\mathrm{h}^{2}\right)=32 \mathrm{~h}^{2} / \mathrm{Np}$, em que:

$\mathrm{N}=$ número de indivíduos por famílias de meios-irmãos

$\mathrm{p}=$ número de famílias de meios-irmãos

Para um número total $(\mathrm{Np})$ fixo de indivíduos (tomados aleatoriamente na população não selecionada e com iguais números de indivíduos por família), a variância é minimizada (por diferenciação de $\mathrm{V}(\mathrm{h} 2)$ ) quando $\mathrm{N}=4 / \mathrm{h}^{2}+1$ ou aproximadamente $n=4 / h^{2}$ (ROBERTSON, 1959). Assim, o número adequado de indivíduos por família é da ordem de 80, 40, 27, 20, e 13 para herdabilidades da ordem de 0,$05 ; 0,10 ; 0,15 ; 0,20$ e 0,30 , respectivamente. $\mathrm{O}$ número $\mathrm{N}$ adequado é um compromisso entre estimar precisamente a variância dentro de 
famílias ( $\mathrm{N}$ grande) e a variância entre famílias (N pequeno). Os números $\mathrm{N}$ recomendados são menores do que aqueles necessários para a maximização da acurácia seletiva (RESENDE, 1995). Uma vez que os dados experimentais são, em geral, utilizados tanto para o propósito de estimação de parâmetros genéticos, quanto para a seleção, os valores de $\mathrm{N}$ utilizados na prática são maiores que os recomendados. Este fato não é problemático devido ao fato de discrepâncias de $\mathrm{N}$ em relação ao ótimo terem pequeno efeito na eficiência da estimação, visto que o delineamento de meios-irmãos é robusto, exceto quando as discrepâncias forem para valores menores do que o indicado (THOMPSON, 1989).

$\mathrm{O}$ número total $\mathrm{T}=\mathrm{Np}$ de indivíduos pode ser determinado escolhendo-se a priori um valor desejado do desvio padrão da herdabilidade, sendo dado por $\mathrm{T}=\left[32 \mathrm{~h}^{2} / \operatorname{Var}\left(\mathrm{h}^{2}\right)\right]^{1 / 2}$

\section{Estimação da herdabilidade em progênies de A.mearnsii}

O estudo foi realizado a partir de experimentação realizada com 43 progênies de meios-irmãos oriundas de populações naturais de A. mearnsii

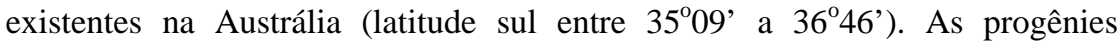
testadas foram colhidas, em 1985, pelo CSIRO.

O delineamento experimental utilizado na instalação do experimento foi o de blocos casualizados com arranjo hierárquico de famílias dentro de populações, com 10 repetições, sendo cada parcela representada por seis árvores, plantadas em fileira. No experimento foi implantada bordadura dupla, com mudas utilizadas em plantio comercial. As informações geográficas do local do experimento estão na Tabela 1 .

Tabela 1 - Informações geográficas sobre o experimento

Table 1 - Geographical information about the experiment

\begin{tabular}{ccccc}
$\begin{array}{c}\text { Município } \\
\text { do RS }\end{array}$ & Latitude & Longitude & Altitude & Precipitação \\
\hline Cristal & $\mathrm{S}$ & $\mathrm{W}$ & $\mathrm{m}$ & $\mathrm{mm}$ \\
\hline
\end{tabular}

Após o preparo de solo, ou seja, subsolagem e gradagem na linha de plantio, as mudas foram plantadas no espaçamento de $3,0 \mathrm{~m} \times 1,5 \mathrm{~m}$ e receberam 80 gramas de adubo da formulação 5:30:15, aplicados na cova.

Aos 3 anos de idade foram mensurados o DAP, altura e sobrevivência das árvores. Para o estudo optou-se somente pela análise do DAP. 
As estimativas das variâncias e herdabilidades foram obtidas pelo método iterativo da máxima verossimilhança restrita (REML) utilizando o programa DFREML (MEYER, 1998). O modelo utilizado foi:

$$
\mathrm{Y}=\mathrm{Xf}+\mathrm{Za}+\mathrm{Wc}+\mathrm{e}, \text { em que: }
$$

$\mathrm{f}, \mathrm{a}, \mathrm{c}, \mathrm{e}=$ vetores de efeitos fixos (de blocos e de procedências), de valores genéticos aditivos, de parcela e erros aleatórios, respectivamente.

$\mathrm{X}, \mathrm{Z}$ e W = matrizes de incidência para $\mathrm{f}$, a e c, respectivamente.

As equações de modelo misto associados ao modelo são (RESENDE, 1999):

$$
\begin{aligned}
& {\left[\begin{array}{c}
\hat{b} \\
\hat{a} \\
\hat{c}
\end{array}\right]=\left[\begin{array}{ccc}
X^{\prime} X & X^{\prime} Z & X^{\prime} W \\
Z^{\prime} X & Z^{\prime} Z+A^{-1} \lambda & Z^{\prime} W \\
W^{\prime} X & W^{\prime} Z & W^{\prime} W+I \lambda_{2}
\end{array}\right]^{-1}\left[\begin{array}{c}
X^{\prime} Y \\
Z^{\prime} Y \\
W^{\prime} Y
\end{array}\right]} \\
& \lambda_{1}=\frac{1-h^{2}-c^{2}}{h^{2}} ; \quad \lambda_{2}=\frac{1-h^{2}-c^{2}}{c^{2}}
\end{aligned}
$$

em que:

$\mathrm{h}^{2}=$ herdabilidade individual no sentido restrito

$\mathrm{c}^{2}=$ correlação intraclasse devida ao ambiente comum da parcela

Os componentes de variância e parâmetros genéticos foram estimados a partir de subconjuntos de dados de diferentes tamanhos utilizando-se 3 (6 combinações diferentes), 6 (4 combinações diferentes) e 10 blocos.

\section{RESULTADOS E DISCUSSÃO}

\section{Minimização da variância da estimativa da herdabilidade}

Na Tabela 2 são apresentadas as magnitudes adequadas de T, N e p para alguns valores de herdabilidade e desvios padrões desejados.

Observa-se que, para se obter o desvio padrão equivalendo a $50 \%$ do valor da herdabilidade, em caracteres de baixa herdabilidade $(<0,30)$, o número adequado de indivíduos é relativamente pequeno e aumenta a medida que $a h^{2}$ diminui. Para se obter um valor fixo (invariável em valor absoluto, para todas as herdabilidades) de $\left[\operatorname{Var}\left(\mathrm{h}^{2}\right)\right]^{1 / 2}$, caracteres com maiores herdabilidades exigem maiores números de indivíduos e de famílias em relação aos caracteres 
com mais baixa $\mathrm{h}^{2}$. Para se obter $\left[\operatorname{Var}\left(\mathrm{h}^{2}\right)\right]^{1 / 2}$ igual a $20 \%$ das estimativas, $\mathrm{N}$ da ordem de 2600 a 8000 são necessários para $\mathrm{h}^{2}$ de 0,30 a 0,10 , respectivamente.

Quanto à precisão (desvio padrão) necessária nos procedimentos de predição de valores genéticos tem sido relatado que o uso de índices combinados baseados em informação de família são robustos a erros na herdabilidade, visto que pouco progresso genético é perdido quando se usam herdabilidades incorretas. Por outro lado, índices baseados em vários caracteres são menos robustos (SALES \& HILL, 1976 a e b; THOMPSON, 1989). Uma alternativa poderia ser a consideração dos intervalos de confiança na faixa de $10 \%$ (desvios padrões de 0,05 ) para as $\mathrm{h}^{2}$ onde os intervalos de 0,05 a 0,15 ; 0,10 a 0,$20 ; 0,15$ a 0,$25 ; 0,20$ a 0,30 e 0,25 a 0,35 conteriam as herdabilidades de 0,$10 ; 0,15 ; 0,20 ; 0,25$ e 0,30 , respectivamente. Neste caso, os números de indivíduos adequados estariam na faixa de 1200 a 3800, conforme o terço médio da Tabela 2.

Tabela 2 - Números adequados de famílias de meios-irmãos (p), de filhos por genitor ou tamanho de famílias de meios-irmãos $(\mathrm{N})$ e população experimental total $(\mathrm{T}=\mathrm{Np})$ para se obter estimativas de herdabilidade $\left(\mathrm{h}^{2}\right)$ com um determinado desvio padrão $\left.\left[\operatorname{Var}\left(\mathrm{h}^{2}\right)\right]^{1 / 2}\right]$

Table 2 - Adequate numbers of half-sib families ( $p$ ); of offspring per parent or size of half-sib families $(N)$ and total experimental populations $(T=N p)$ for obtaining heritability estimates $\left(h^{2}\right)$ with a standard deviation of $\left.\left[\operatorname{Var}\left(h^{2}\right)\right]^{1 / 2}\right]$

\begin{tabular}{cccccc}
\hline $\mathrm{h}^{2}$ & Situação & {$\left[\operatorname{Var}\left(\mathrm{h}^{2}\right)\right]^{1 / 2}$} & $\mathrm{~T}$ & $\mathrm{~N}$ & $\mathrm{p}$ \\
\hline 0,05 & & 0,025 & 2560 & 80 & 32 \\
0,10 & Desvio & 0,050 & 1280 & 40 & 32 \\
0,15 & padrão & 0,075 & 853 & 27 & 32 \\
0,20 & equivalendo & 0,100 & 640 & 20 & 32 \\
0,25 & a 50\% de $\mathrm{h}^{2}$ & 0,125 & 512 & 16 & 32 \\
0,30 & & 0,150 & 426 & 13 & 32 \\
0,05 & & 0,05 & 640 & 80 & 8 \\
0,10 & & 0,05 & 1280 & 40 & 32 \\
0,15 & Valor fixo de $_{0,05}$ & 1920 & 27 & 72 \\
0,20 & Var(h $\left.\left.^{2}\right)\right]^{1 / 2}$ & 0,05 & 2560 & 20 & 128 \\
0,25 & igual a 0,05 & 0,05 & 3200 & 16 & 200 \\
0,30 & & 0,05 & 3840 & 13 & 295 \\
0,05 & & 0,01 & 16000 & 80 & 200 \\
0,10 & [Var(h $\left.\left.^{2}\right)\right]^{1 / 2}$ & 0,02 & 8000 & 40 & 200 \\
0,15 & igual & 0,03 & 5333 & 27 & 200 \\
0,20 & ${\text { a 20\% de } h^{2}}_{0,25}$ & 0,04 & 4000 & 20 & 200 \\
0,30 & & 0,05 & 3200 & 16 & 200 \\
\hline & & 2666 & 13 & 200 \\
\hline
\end{tabular}


Por outro lado, considerando $\mathrm{N}$ fixo igual a 1000, seriam obtidos os seguintes intervalos de confiança: 0,01 a 0,$09 ; 0,04$ a 0,$16 ; 0,08$ a 0,22 e 0,12 a 0,28 para herdabilidades de 0,$05 ; 0,10 ; 0,15$ e 0,20 e p de $13 ; 25 ; 37$ e 50 famílias, respectivamente. Assim, pode-se inferir que pelo menos cerca de 1000 dados e mais do que 20 famílias deveriam ser utilizados na estimação de parâmetros genéticos. O tamanho amostral de 1000 foi recomendado também como valor mínimo para estimação de parâmetros genéticos com vistas a construção de índices de seleção envolvendo caracteres com herdabilidade menores ou igual a 0,20 (HARRIS, 1964).

\section{Resultados experimentais relativos à herdabilidade em acácia-negra}

Em estudos conduzidos no Brasil e na China, com progênies de $A$. mearnsii, as estimativas da herdabilidade individual para o caráter DAP variaram de 0,29 a 0,37 , com média 0,33 (Tabela 3).

Tabela 3 - Estimativas de herdabilidade para o caráter DAP obtidas de literatura Table 3 - Heritability estimates for the trait DAP obtained from literature

\begin{tabular}{lccccc}
\hline Autor & $\begin{array}{c}\text { Número de } \\
\text { Progênies }\end{array}$ & $\begin{array}{c}\text { Número de } \\
\text { Repetições }\end{array}$ & $\begin{array}{c}\text { Árvores por } \\
\text { parcela }\end{array}$ & Idade & $\begin{array}{c}\text { DAP } \\
\mathrm{h}^{2} \text { indivíduo }\end{array}$ \\
\hline Resende et al.1992 & 46 & 5 & 5 & 3 & 0,31 \\
Resende et al.1992 & 46 & 5 & 5 & 3 & 0,36 \\
Bi et al., 1991 & 169 & 20 & 1 & 2 & 0,29 \\
Bi et al., 1991 & 169 & 20 & 1 & 2 & 0,37 \\
\hline \multicolumn{1}{c}{ Média } & & & & & 0,33 \\
\hline
\end{tabular}

Aos 3 anos de idade, a sobrevivência das árvores nos dez blocos foi de $81,3 \%$, ou seja, das 2580 mudas plantadas, 2099 foram avaliadas. Na Tabela 4 são apresentadas as estimativas dos parâmetros genéticos e fenotípicos associados ao experimento de acácia-negra. 
Tabela 4 - Valores encontrados de média, variâncias, herdabilidade e desvio padrão da herdabilidade em função do número de repetições utilizados

Table 4 - Average, heritability and standard deviation values obtained as a function of replications number used

\begin{tabular}{lccccccc}
\hline \multicolumn{1}{c}{ BLOCOS } & $\mathrm{T}$ & Média & $\begin{array}{c}\text { Desvio } \\
\text { padrão }\end{array}$ & $\begin{array}{c}\text { Variância } \\
\text { Fenotípica }\end{array}$ & $\begin{array}{c}\text { Variância } \\
\text { Aditiva }\end{array}$ & $\mathrm{h}^{2}$ & [Var(h $\left.^{2}\right]^{1 / 2}$ \\
\hline 1 a 10 & 2099 & 8.02 & 1.99 & 3.8627 & 1.2981 & 0.3345 & 0,0714 \\
\hline 1, 2, 3, 4, 5 e 6 & 1269 & 7.99 & 2.00 & 3.9530 & 1.4431 & 0.3651 & 0,0959 \\
1, 2, 4, 5, 7 e 8 & 1284 & 8.05 & 1.96 & 3.8268 & 1.5564 & 0.4067 & 0,1006 \\
4, 5, 6, 7, 8 e 9 & 1257 & 8.10 & 1.98 & 3.7795 & 1.0328 & 0.2733 & 0,0834 \\
2, 3, 5, 6, 8 e 9 & 1262 & 8.13 & 2.00 & 3.8479 & 0.9264 & 0.2408 & 0,0781 \\
\hline média & 1268 & 8.07 & 1.99 & 3.8518 & 1.2397 & 0.3215 & 0.0895 \\
\hline CV\% & $1 \%$ & $1 \%$ & $1 \%$ & $2 \%$ & $25 \%$ & $24 \%$ & $12 \%$ \\
\hline 1, 2 e 3 & 633 & 7.98 & 1.99 & 3.9760 & 1.3500 & 0.3394 & 0,1309 \\
4, 5 e 6 & 636 & 8.00 & 2.01 & 3.9305 & 0.9337 & 0.2376 & 0,1093 \\
7, 8 e 9 & 621 & 8.20 & 1.95 & 3.6284 & 0.8293 & 0.2285 & 0,1085 \\
1, 4 e 7 & 628 & 7.94 & 1.94 & 3.8242 & 1.7999 & 0.4707 & 0,1548 \\
2, 5 e 8 & 656 & 8.15 & 1.97 & 3.8216 & 1.1925 & 0.3121 & 0,1233 \\
3, 6 e 9 & 606 & 8.09 & 2.04 & 3.9026 & 1.0573 & 0.2709 & 0,1196 \\
\hline média & 629 & 8.06 & 1.98 & 3.8472 & 1.1938 & 0.3099 & 0.1244 \\
\hline CV\% & $3 \%$ & $1 \%$ & $2 \%$ & $3 \%$ & $29 \%$ & $29 \%$ & $14 \%$ \\
\hline
\end{tabular}


Figura 1 - Valores de herdabilidades em função do número de repetições analisadas Figure 1 - Heritability values as a function of the replications number used

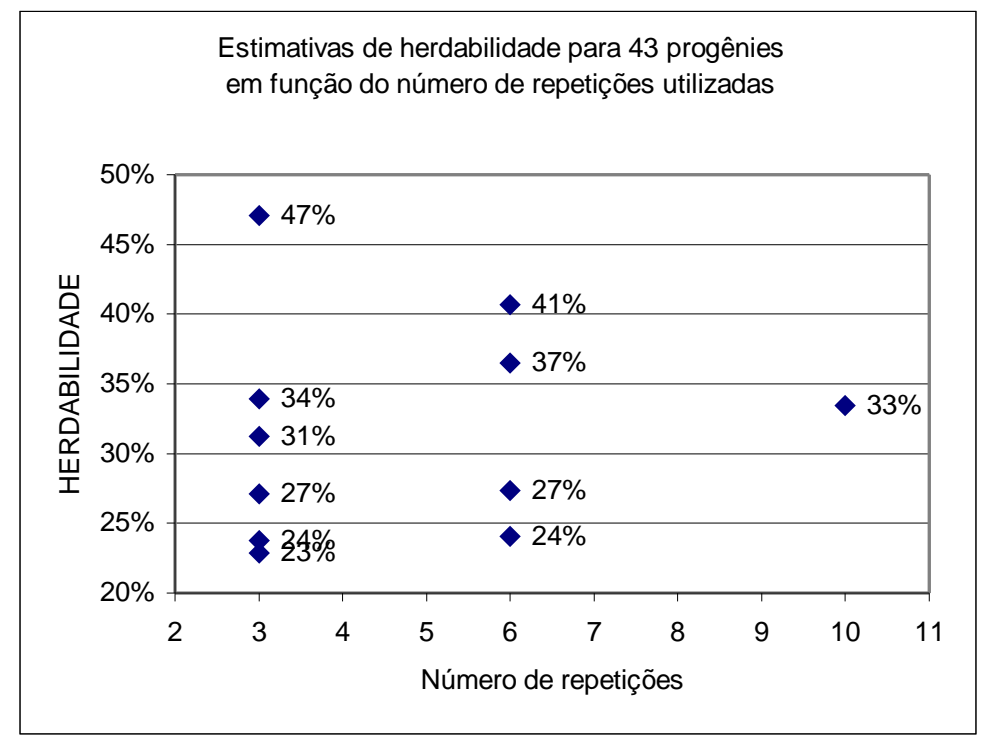

Verifica-se que a média e a variância fenotípica foram bem estimadas com 3, 6 ou 10 repetições. Por outro lado, as estimativas da variância aditiva e da herdabilidade oscilaram bastante quando foram utilizadas 3 e 6 repetições (Tabela 4). Constata-se também que os desvios-padrões da $h^{2}$ foram muito maiores (proporcionalmente) quando obtidos com apenas 3 repetições, conforme esperado teoricamente (Tabela 2).

A estimativa mais fidedigna da herdabilidade foi de $0,33+0,07$, obtida com base em 2099 plantas (10 repetições) das 43 progênies. O valor médio das estimativas obtidas com base em 6 repetições (em torno de 1268 plantas) foi de 0,32 e a média das estimativas obtidas com base em 3 repetições (em torno de 629 plantas) foi de 0,31 . Considerando a média das estimativas obtidas de literatura $(0,33)$ e os resultados obtidos no presente trabalho, pode-se inferir que um valor plausível da herdabilidade para o DAP em acácia-negra situa-se ao redor de 0,30 .

Com base nos resultados da Tabela 4 e na Figura 1, pode-se inferir que estimativas individuais da $\mathrm{h}^{2}$ obtidas com base em 3 e 6 repetições são inadequadas pois conduziriam a valores bem discrepantes do valor mais provável. Dessa forma, para a estimação fidedigna da herdabilidade devem ser considerados (pelo menos aproximadamente) os tamanhos amostrais 
recomendados. Considerando o tamanho de progênie geralmente adotado na prática do melhoramento florestal (6 plantas por parcela e 10 repetições), números esses que maximizam a acurácia seletiva, conforme RESENDE (1995), são apresentadas na Tabela 5, os tamanhos amostrais recomendados para a estimação da herdabilidade, bem como o número de famílias associado.

Tabela 5 - Tamanho amostral $(\mathrm{T})$ aproximado, número de plantas por família $(\mathrm{N}=60)$ e número de família (p) adequados a estimação da herdabilidade $\left(\mathrm{h}^{2}\right)$ com razoável precisão $\left[\mathrm{s}\left(\mathrm{h}^{2}\right)\right]$

Table 5 - Approximated sample size $(T)$, number of plants per family $(N=60)$ and family number $(p)$ adequate for heritability $\left(h^{2}\right)$ estimation with reasonable precision $\left[s\left(h^{2}\right)\right]$

\begin{tabular}{ccccc}
\hline $\mathrm{h}^{2}$ & $\left(\mathrm{~s}\left(\mathrm{~h}^{2}\right)\right)$ & $\mathrm{T}$ & $\mathrm{N}$ & $\mathrm{p}$ \\
\hline 0,05 & 0,01 & 16000 & 60 & 267 \\
0,10 & 0,02 & 8000 & 60 & 133 \\
0,15 & 0,03 & 5400 & 60 & 90 \\
0,20 & 0,04 & 4000 & 60 & 67 \\
0,25 & 0,05 & 3200 & 60 & 53 \\
0,30 & 0,06 & 2600 & 60 & 43 \\
0,40 & 0,08 & 2000 & 60 & 33 \\
0,50 & 0,10 & 1600 & 60 & 27 \\
0,60 & 0,12 & 1400 & 60 & 23 \\
\hline
\end{tabular}

Os valores de $\mathrm{T}$ apresentados na Tabela 5 reforçam a inferência de que quando o número total de dados for inferior a 1000 não se recomenda utilizálos para a estimação de parâmetros genéticos. Neste caso, a predição de valores genéticos deve ser realizada utilizando-se valores médios das herdabilidades obtidas na literatura. McCUTCHAN (1986) também concluiu que ao menos 1000 observações, associadas a uma sobrevivência de pelo menos $80 \%$, são necessárias para a estimação da $h^{2}$.

Para a estimação da herdabilidade no sentido amplo $\left(h_{a}^{2}\right)$, tem-se que $\operatorname{Var}\left(h_{a}^{2}\right)=8 h_{a}^{2} / N$. Verifica-se por esta expressão que para herdabilidades e precisões similares, a estimação de $h_{a}^{2}$ requer menor número $(\mathrm{N})$ total de indivíduos. Com $\mathrm{n}=15$ repetições por clone, pode-se inferir que são necessários 17 clones para que $h_{a}^{2}$ seja estimada com razoável precisão (similar àquela obtida com $\mathrm{N}=1000$ indivíduos em estrutura de famílias de meios-irmãos, para a estimação de $\mathrm{h}^{2}$ no sentido restrito). 


\section{CONCLUSÕES}

Para uma precisa estimação da herdabilidade no sentido restrito recomenda-se os tamanhos amostrais aproximados de 8000, 4000 e 2600 dados para herdabilidades de 0,$10 ; 0,20$ e 0,30 respectivamente.

O valor mais provável para a herdabilidade do caráter DAP em acácianegra situa-se ao redor de 0,30, na idade de 3 anos.

Para estimação da média e variância fenotípica de um caráter quantitativo, a utilização de um tamanho amostral equivalendo a cerca de $1 / 3$ do tamanho amostral adequado à estimação da variância aditiva e da herdabilidade já é suficiente para a obtenção de estimativas precisas.

Com tamanho amostral inferior a 1000 não se recomenda a estimação de parâmetro genéticos. Neste caso, a predição de valores genéticos deve ser realizada preferencialmente utilizando estimativas de parâmetros relatadas em literatura.

Para a estimação adequada da herdabilidade no sentido amplo, pelo menos 250 rametes (por exemplo, 17 clones com 15 rametes de cada) são necessários.

\section{BIBLIOGRAFIA CITADA}

BI, G. C.; YUAN, L. J.; WILlIAMS, E. R. Performance of Acacia mearnsii provenances / progeny in Southern China. In: TURNBULL, J. W. Advances in tropical acacia research. ACIAR Proceedings n. 35, p. 215-218, 1991.

BORÉM, A. Melhoramento de plantas. 2.ed. Viçosa. UFV. 453p.

CASTRO, N.H.C. de Número de repetições e eficiência da seleção em progênies de meios-irmãos de Eucalyptus camaldulensis. ESALQ. Lavras. Dissertação de mestrado. 96p. 1992.

COTERILL, P.P. JAMES, J. Number of offspring and plot sizes required for progeny testing. Silvae Genetica, v.23, n.6, p.203-8, 1984.

HAAPANEN, M. Effect of plot size and shape on the efficiency of progeny tests. Silva Fennica, v.26, n.4, p.201-9, 1992.

HARRIS, D. L. Expected and predicted progress from index selection involving estimates of population parameters. Biometrics, v.20, p.46-72, 1964. 
HILL.W.G. Considerations in the design of animal breeding experiments. In: Gianola, D. Hammond, K.(eds) Advances in statistical methods for genetic improvement of livestock. Spring-Verlag, Berlin, p. 59 -76, 1990.

LAMBETH, C; GLADSTONE, W.; STONECYPHER, R. Statistical efficiency of row and noncontiguous family plots in genetic tests of loblolly pine. Silvae Genetica, v.32, n. 1-2, p.24-8, 1983.

LEE, C. H. Statistical efficiency varies with plot size, number of replications and seedlots sampled, Silvae Genetica, v.32, p.49-52, 1983.

McCUTCHAN, B.G. Design efficiencies with planned and unbalance for estimating heritability in forestry. In: Southern Forest Tree Improvement Conference, 18. Mississippi, 1986, p.117-126.

MEYER, K. DFREML - Version 3.0 User Notes. Institute of Animal Genetics of Edinburgh-Scotland / Animal Genetics and Breeding Unit of the University of New England, Armidale - Austrália. 1998. 31p.

MIRANDA FILHO, J.B. Princípios de experimentação e análise estatística. In: Paterniani, E. (Ed.) Melhoramento e produção do milho no Brasil Marprint, Piracicaba, p.621-649.

RESENDE, M.D.V. de. Predição de valores genéticos, componentes de variância, delineamentos de cruzamento e estrutura de populações no melhoramento florestal. Curitiba, 1999. 434p. (Tese Doutorado).

RESENDE, M. D. V. de et al. Parâmetros genéticos e interação genótipo x ambiente em teste de procedência e progênies de acácia-negra (Acacia mearnsii De Wild). Boletim Pesquisa Florestal, Colombo. 24/25. p.55-65. 1992

RESENDE, M. D. V. de . Delineamento de experimentos de seleção para a maximização da acurácia seletiva e do progresso genético. Revista Árvore, v.19, n.4, p.479-500, p.1995.

RESENDE, M.D.V. de; ROSA-PEREZ, J.R.H. Genética Quantitativa e Estatística no Melhoramento Animal. Imprensa Universitária, Universidade Federal do Paraná. Curitiba, 1999. 387p.

ROBERTSON, A. Experimental design in the evaluation of genetic parameters. Biometrics, v.15, p.219-226, 1959. 
SALES, HILL, W.G. Effect of sampling errors on efficiency of selection indices. 2 Use of information from relatives for single trait improvement. Animal Production, v.22, p,1-17, 1976a.

SALES, HILL, W.G. Effect of sampling errors on efficiency of selection indices. 2 Use of information on associated traits for improvement of a single trait. Animal Production, v.23, p,1-14, 1976b.

THOMPSON, R. Design of experiments to estimate genetic parameters within populations . In: HILL, W.G.; MACKAY,T. (Eds.). Evolution and animal breeding. Edinburgh: CAB international, 1989. P.169-74.

WOOLONS, R.C. Importance of experimental design and plot maintenance in forest field experiments in Australasia. Australian Forest Research, v.10, p.71-82, 1980.

WRIGHT, J.M.; FREELAND, F.D. Plot size and experimental efficiency in forest genetics research. S.e. S.n., 1960 27p. (Agr. Exp. Stat. Tech. Bull, 280). 